\title{
Comparação entre o xenodiagnóstico clássico e artificial na fase crônica da doença de Chagas
}

\author{
Comparison between the classical and artificial xenodiagnosis in \\ chronical Chagas' disease
}

\author{
Jorge Panameño Pineda, Alejandro Luquetti e Cleudson Costro
}

\begin{abstract}
Resumo $O$ xenodiagnóstico (xeno) clássico e artificial feitos com Dipatalogaster maximus de primeiro estágio foi realizado simultaneamente em 57 pacientes com infecção chagásica crônica (22 do sexo masculino e 35 do sexo feminino, com idades entre 7 e 80 anos). Exceto dois pacientes com clínica de megaesôfago, os demais tinham dois exames sorológicos prévios positivos sendo feita nova sorológia no decorrer do estudo. Os pacientes eram provenientes do ambulatório do Hospital Universitário de Brasília (HUB) ou eram residentes no município de Mambaí, GO. Dos 57 pacientes, 24 (42\%) apresentaram xenodiagnósticos positivos. Dos 114 xenodiagnósticos realizados, 36 (32\%) foram positivos. A comparação das duas técnicas não mostrou diferença estatisticamente significante $(p=0,42)$, porém o xeno artificial apresenta vantagem porque o sangue é oferecido aos triatomíneos através de um aparelho enquanto, no xeno clássico, os triatomíneos sugam através da pele do paciente.
\end{abstract}

Palavras-chaves: Xenodiagnóstico. Xenodiagnóstico artificial. Doença de Chagas.

\begin{abstract}
Classical and artificial xenodiagnostic techniques made with Dipetalogaster maximus of first stage were performed simultaneously in 57 patients with chronic T. cruzi infection (22 male and 35 female patients, aged 7-80 years). With the exception of two patients with megaoesophagus, all had two previous positive serological reaction and a further test was done at the time of the examination. The patients came from the outpatient department of the university hospital or were resident in Mambaí, Goiás. Of the 57 patients, 24 (42\%) had a positive xenodiagnoses. Of a total of 114 tests performed, 36(32\%) were positive. Comparing the two xenodiagnostic techniques, no significant advantage was apparent statistically $(p=0,42)$, but the artificial technique has advantages because the blood is offered for triatomines through a device while in the classical technique, the triatomines suck through the patient's skin.
\end{abstract}

Key-words: Xenodiagnosis. Artificial xenodiagnosis. Chagas'disease.

Núcleo de Medicina Tropical e Nutrição, Universidade de Brasília, Brasília DF e Faculdade de Medicina da Universidade Federal de Goiás GO, Brasil

Endereço para correspondência: Prof. Cleudson Castro. Núcleo de Medicina Tropical e Nutrição/UnB. Caixa Postal 04671, 70919-970 Brasília DF, Brasil.

Fax: (061) 273-2811

Recebido para publicação em 11/08/97. 
O xenodiagnóstico continua sendo o melhor método para o diagnóstico parasitológico na fase crônica da doença de Chagas. Foi introduzido por Brumpt1, após observar o desenvolvimento de tripanossomas de peixes no tubo digestivo de sanguessugas e de isolar o T. cruzi de uma cobaia usando o então denominado Triatoma megista. Após seus experimentos feitos em Paris 19 , houve um largo silêncio na literatura. $\mathrm{O}$ método foi usado pela primeira vez no ser humano por Margarinos Torres e, em 1933, por Torrealba que encontrou $25 \%$ de chagásicos entre os colonos de Guárico 18 19. Dias 5 foi o primeiro a estabelecer critérios para a utilização deste método, especialmente no que diz respeito ao triatomíneo a ser utilizado. No xenodiagnóstico clássico, os triatomíneos têm contato direto com a pele do paciente, e pode causar irritação local com surgimento de máculas ou pápulas pruriginosas e, raramente, choque anafilático. Devido a isto, Romaña e Gil11 introduziram, na década de 40, o xenodiagnóstico artificial. Este método não foi usado na rotina durante anos mas, ultimamente, tem-se dado grande ênfase a ele principalmente por causa da reativação da doença de Chagas em cardiopatas transplantados, e em imunossuprimidos, principalmente aidéticos. Este trabalho tem como finalidade comparar o xenodiagnóstico clássico com o artificial e avaliar sua aplicação na zona rural.

\section{MATERIAL E MÉTODOS}

População estudada. O trabalho foi realizado em chagásicos crônicos procedentes do ambulatório do Hospital Universitário de Brasília e do Município de Mambaí, Estado de Goiás, onde a doença de Chagas é endêmica e, em um grupo controle não chagásico. Todos os chagásicos, exceto dois, tinham pelo menos duas diferentes reações sorológicas prévias positivas e foram admitidos no estudo independente da forma clínica. Os dois pacientes sem sorologia prévia foram admitidos com base na epidemiologia e na clínica de megaesôfago. Todos eram virgens de tratamento exceto um que usou benznidazol, mas persistia com parasitemia. O grupo controle foi constituído por funcionários, professores e estudantes de medicina da Universidade de Brasília, com epidemiologia e sorologia supostamente negativas e também por pacientes de Mambaí com sorologia sabidamente negativa para infecção chagásica. Foram realizados em pacientes de Mambaí 12 xenodiagnósticos clássicos e dezenove artificiais.

Foi feito em três pacientes de Mambaí, residentes a $30 \mathrm{~km}$ da sede, o xeno clássico e, ao mesmo tempo, foi coletado sangue para o xeno artificial que foi realizado na sede municipal, quatro horas após.

Estudo xenodiagnóstico. Foram usados, em cada xenodiagnóstico, 40 ninfas do $1^{\circ}$ estágio de Dipetalogaster maximus, procedentes do insetário do Núcleo de Medicina Tropical e Nutrição e do laboratório de xenodiagnóstico do Instituto de Patologia Tropical e Saúde Pública da Universidade Federal de Goiás. Em cada chagásico aplicou-se um xenodiagnóstico clássico e, simultaneamente, foi coletado sangue em tubo com heparina para o xenodiagnóstico artificial. Os pacientes foram entrevistados e informados sobre os objetivos e procedimentos do estudo, sendo preenchida uma ficha com dados clínicos e epidemiológicos e, assinaram um termo de consentimento.

Para a realização do xenodiagnóstico clássico, fez-se assepsia de uma das panturrilhas do paciente com algodão embebido em álcool. Após a evaporação foram aplicados durante 30 minutos os triatomíneos contidos em quatro caixas de madeira, cada uma com dez ninfas.

Para a realização do xenodiagnóstico artificial, foi construído um aparelho semelhante ao de Silva16, constituído por uma caixa metálica com reservatório para 3 litros de água, munido com interruptores para aquece-la e faze-la circular. $\mathrm{O}$ aparelho é provido de uma bomba acionada por motor elétrico marca Eberle de 1/40 HP de potência, que propulsiona água aquecida a $37^{\circ} \mathrm{C}$ através de um circuito de mangueiras e artefatos de vidro, conforme a Figura 1. O artefato de vidro em forma de sino tem duas câmaras, uma externa para a circulação da água e outra interna, completada na parte inferior por um preservativo sem lubrificante que serve como reservatório para o sangue e, simultaneamente, como membrana através da qual os triatomíneos sugam. Uma estante de madeira sustenta as mangueiras os artefatos de vidro e os frascos plásticos com os triatomíneos.

Técnica do xenodiagnóstico artificial. Para realização deste exame foram colhidos em veia do braço com agulha multiuso, $12 \mathrm{ml}$ de sangue em tubos vacutainer marca Vacuum II, sendo $7 \mathrm{ml}$ para o xenodiagnóstico artificial e $5 \mathrm{ml}$ para a sorologia de controle. O sangue para a sorologia 


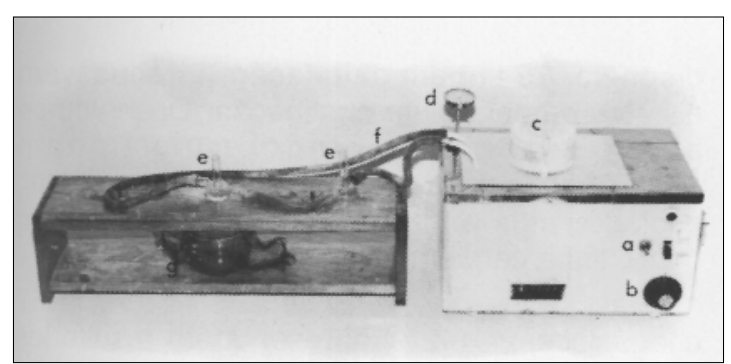

Figura 1 - Aparelho para xenodiagnóstico artificial a) interruptores para aquecer e circular água, b) termostato; c) motor elétrico; d) termômetro, e) mamadeiras de vidro; f) tubulação para circulação de água; g) frasco com triatomíneos.

foi colhido em tubo sem anticoagulante e, o utilizado para o xenodiagnóstico artificial, em tubo contendo 20,4UI de heparina sódica para cada $\mathrm{ml}$ de sangue. A seguir, o sangue heparinizado foi colocado na câmara interna do artefato de vidro, ficando retido no preservativo de látex. Um frasco plástico, vedado por filó, contendo os triatomíneos foi posto na estante de modo que os insetos entravam em contato com o sangue através do preservativo e sugavam durante 30 minutos.

Leituras dos xenodiagnósticos. realizado o xenodiagnóstico, os triatomíneos foram mantidos à temperatura ambiente, em estante apropriada, até o momento da leitura. Só foram examinados os triatomíneos que mudaram de estágio. Para o preparo das lâminas, os insetos foram colocados em um copo de vidro e manipulados por um técnico diferente do microscopista, de modo que este não sabia se estava lendo um xenodiagnóstico clássico, artificial ou controle. As leituras foram praticadas 45 dias após a aplicação dos xenodiagnósticos sendo realizada a dissecção intestinal dos triatomíneos. Cada lâmina foi preparada com um pool do conteúdo intestinal de cinco triatomíneos, exceto a última lâmina, geralmente constituída com um pool de menos de cinco insetos. $O$ intestino foi macerado sobre a lâmina, diluído em $0,05 \mathrm{ml}$ de soro fisiológico e coberto com lamínula de $32 \mathrm{x}$ $24 \mathrm{~mm}$. As lâminas foram examinadas com um aumento de 100x e, em caso de dúvida, de 400x até o encontro do T. cruzi. Quando negativas, foram examinadas em toda a extensão, durante 15 minutos.

Sorologia prévia e sorologia de controle. Antes de iniciar-se o estudo, foi verificado o resultado da sorologia previamente realizada.
Para os pacientes do HUB, a sorologia foi verificada nos prontuários $e$, para os chagásicos de Mambaí, foi tomada do livro de registro do projeto. Exceto dois, os demais tinham pelo menos duas sorologias positivas pelas seguintes técnicas: imunofluorescência indireta (IFI), hemaglutinação indireta (HAI), fixação do complemento (FC) ou ELISA. Para confirmar a sorologia prévia do grupo chagásico e a suposta negatividade dos indivíduos do grupo controle, foi realizada a sorologia de controle. Para isto, foi colhido cinco mililitros de sangue que, após coagulado, foi centrifugado, separado o soro e armazenado em freezer a $-20^{\circ} \mathrm{C}$ e, posteriormente, transportado para Goiânia acondicionado em caixa de isopor contendo gelo. A sorologia foi processada no laboratório de pesquisa em doença de Chagas do Hospital das Clínicas da Universidade Federal de Goiás, através das reações de HAI, IFI e ELISA.

Análise estatística. Os dados foram processados através do programa SAS 248 versão 1994 (SAS Institute Inc. 1994). Foram utilizados os testes do qui quadrado ou o teste exato de Fisher, sendo considerados significativos os valores de $p$ menores que 0,05 .

\section{RESULTADOS}

Foram examinados 57 chagásicos, dos quais $22(38,6 \%)$ eram do sexo masculino e 35 $(61,4 \%)$ do sexo feminino. A idade variou entre 7 a 80 anos, sendo a média de 38 anos. A faixa etária com mais indivíduos foi a de 31 a 60 anos com 36 pacientes (Tabela 1). Foram examinados, também, 21 controles, soronegativos para infecção chagásica, sendo 11 do sexo masculino e 10 do feminino. No total, foram estudados 78 indivíduos.

Tabela 1 - Distribuição dos chagásicos, de acordo com a faixa etária e sexo.

\begin{tabular}{lccc}
\hline Faixa etária & Masculino & Feminino & Total \\
\hline $07-30$ & 4 & 9 & 13 \\
$31-60$ & 13 & 23 & 36 \\
$61-80$ & 5 & 3 & 8 \\
\hline Total & 22 & 35 & 57 \\
\hline
\end{tabular}

Os 57 chagásicos eram provenientes de nove Estados brasileiros sendo 21 de Goiás, 18 de Minas Gerais, 8 da Bahia, 3 do Distrito Federal, 2 do Piauí, 2 da Paraíba e 1 dos Estados 
de Pernambuco, Ceará e Tocantins. Foram estudados 45 indivíduos em Brasília, DF e 12 em Mambaí, GO.

Em relação às formas clínicas dos 57 chagásicos, $19(33,3 \%)$ eram da forma cardíaca, $11(19,3 \%)$ da digestiva, $15(26,4 \%)$ da mista (cardíaca + digestiva) e $12(21 \%)$ da forma indeterminada. Dos cardiopatas, três apresentavam cadiomegalia ao Rx de tórax, dez referiam dispnéia de esforço e oito tinham palpitações. Entre os que apresentavam a forma digestiva, dez referiam disfagia e quatro obstipação. Sete e três tinham respectivamente esofagograma e clister opaco alterados. Dos portadores da forma mista, seis apresentavam dispnéia, 15 tinham palpitação, 11 acusavam disfagia e apenas um obstipação. Observou-se ainda que cinco, cinco e um tinham respectivamente eletrocardiograma, esofagograma e clister opaco alterados e, um outro, tinha cardiomegalia ao $\mathrm{Rx}$ de torax.

Resultados dos xenodiagnósticos. Dos 57 chagásicos, 24 (42\%) apresentaram resultados positivos e dos 114 xenos neles realizados 36 (32\%) foram positivos (Tabelas 2 e 3). Analisando os xenodiagnósticos clássico e artificial viu-se que $16(28 \%)$ e $20(35 \%)$, respectivamente, apresentaram resultados positivos, não havendo diferença estatisticamente significante $(p=0,42)$, Tabela 2.

Tabela 2 - Resultados dos xenodiagnósticos clássico e artificial aplicados em 57 chagásicos crônicos

\begin{tabular}{|c|c|c|c|c|c|c|}
\hline \multirow[t]{2}{*}{ Xenodiagnóstico } & \multicolumn{2}{|c|}{ Positivos } & \multicolumn{2}{|c|}{ Negativos } & \multicolumn{2}{|c|}{ Total } \\
\hline & $\mathrm{n}^{\circ}$ & $\%$ & $\mathrm{n}^{\circ}$ & $\%$ & $\mathrm{n}^{\circ}$ & $\%$ \\
\hline Clássico & 16 & $28^{*}$ & 41 & 72 & 57 & 100 \\
\hline Artificial & 20 & $35^{\star}$ & 37 & 65 & 57 & 100 \\
\hline Total & 36 & 32 & 78 & 68 & 114 & 100 \\
\hline
\end{tabular}

${ }^{*} p=0,42$

Tabela 3 - Distribuição dos chagásicos segundo o resultado do xeno clássico, artificial e com ambos os métodos.

\begin{tabular}{lcccc}
\hline Resultados & Xeno clássico & Xeno artificial & Ambos os métodos & Total \\
\hline Positivos & 4 & 8 & 24 & 21 \\
Negativos & 4 & 8 & 33 \\
\hline Total & 8 & 16 & 53
\end{tabular}

Dos 57 chagásicos, 4 (7\%) foram positivos somente pelo método natural, $8(14 \%)$ apenas pelo artificial e $12(21 \%)$ foram positivos por ambos os métodos, Tabela 3.

A distribuição dos 24 chagásicos xenopositivos pelos métodos clássico e/ou artificial está mostrada na Tabela 4, onde se observa a relação de pools positivos sobre os pools examinados. A tabela mostra que no xeno clássico houve $30,1 \%(53 / 176)$ de pools positivos e, no xeno artificial, 24,4\% (44/180). Dos 16 que apresentaram xenos positivos pelo método clássico, $6(37,5 \%)$ foram do sexo masculino e $10(62,5 \%)$ do sexo feminino. Dos 20 pacientes positivos pelo método artificial, 7 (35\%) foram do sexo masculino e 13 (65\%) do sexo feminino.

A distribuição da xenopositividade, de acordo com as faixas etárias, esta mostrada na Tabela 5.
Quantidade de tritomíneos utilizados. Para 114 xenodiagnósticos realizados nos chagásicos, foram empregados 2280 ninfas do $1^{\circ}$ estágio do D. maximus, tanto no xenodiagnóstico clássico quanto no artificial. Pelo método clássico, 2036 $(89 \%)$ ninfas mudaram para o $2^{\circ}$ estágio, enquanto no xenodiagnóstico artificial, o número foi 1993 (87\%) (Tabela 6). Houve 43 ninfas examinadas a mais no método clássico, sem diferença estatisticamente significante, $(p=0,62)$. A média de triatomíneos examinados por xenodiagnóstico no método clássico foi $36( \pm 4)$ $(90 \%)$ e, no método artificial, foi $35( \pm 3)(88 \%)$, não havendo diferença estatisticamente significante $(p=0,50)$

No xenodiagnóstico clássico, os 2036 triatomíneos examinados originaram 426 "pools", dos quais $383(89,9 \%)$ foram constituídos por 5 triatomíneos, $10(2,3 \%)$ por 4 triatomíneos, 11 
Tabela 4 - Distribuição dos 24 chagásicos cujos xenodiagnósticos natural e/ou artificial foram positivos

\begin{tabular}{|c|c|c|c|c|}
\hline Indivíduos & Sexo & Idade & $\begin{array}{c}\text { Xeno clássico } \\
\text { pools positivos/examinados }\end{array}$ & $\begin{array}{c}\text { Xeno artificial } \\
\text { pools positivos/examinados }\end{array}$ \\
\hline AGS & $\mathrm{F}$ & 38 & $1 / 8$ & $0 / 8$ \\
\hline$A A B$ & M & 53 & $0 / 5$ & $1 / 8$ \\
\hline EAS & $\mathrm{F}$ & 30 & $2 / 8$ & $3 / 8$ \\
\hline SPS & M & 49 & $0 / 7$ & $1 / 8$ \\
\hline AMJ & $\mathrm{F}$ & 58 & $7 / 7$ & $3 / 7$ \\
\hline GFB & M & 63 & $1 / 7$ & $3 / 7$ \\
\hline MPS & $\mathrm{F}$ & 57 & $5 / 7$ & $1 / 6$ \\
\hline DAV & $\mathrm{F}$ & 27 & $0 / 8$ & $4 / 7$ \\
\hline DAVF & $\mathrm{F}$ & 7 & $3 / 8$ & $3 / 8$ \\
\hline ER & M & 80 & $8 / 8$ & $6 / 8$ \\
\hline DGL & $\mathrm{F}$ & 42 & $3 / 8$ & $1 / 8$ \\
\hline EJS & $\mathrm{F}$ & 50 & $0 / 8$ & $1 / 8$ \\
\hline JVB & $\mathrm{F}$ & 47 & $2 / 7$ & $0 / 8$ \\
\hline IDR & $\mathrm{F}$ & 27 & $0 / 8$ & $1 / 7$ \\
\hline AAS & M & 63 & $1 / 8$ & $0 / 8$ \\
\hline JBS & $\mathrm{F}$ & 18 & $0 / 8$ & $2 / 7$ \\
\hline AMF & $\mathrm{F}$ & 67 & $0 / 7$ & $1 / 7$ \\
\hline NLU & $\mathrm{F}$ & 48 & $2 / 7$ & $2 / 8$ \\
\hline $\mathrm{HAC}$ & $\mathrm{F}$ & 44 & $5 / 8$ & $3 / 7$ \\
\hline DCV & M & 30 & $5 / 7$ & $3 / 8$ \\
\hline SD & M & 52 & $0 / 6$ & $1 / 7$ \\
\hline $\mathrm{AF}$ & M & 58 & $3 / 7$ & $0 / 8$ \\
\hline ESC & M & 35 & $2 / 7$ & $1 / 7$ \\
\hline JBN & $\mathrm{F}$ & 26 & $3 / 7$ & $3 / 7$ \\
\hline Total & & & $53 / 176$ & $44 / 180$ \\
\hline
\end{tabular}

Tabela 5 - Distribuição dos chagásicos xenopositivos, segundo a faixa etária e o método utilizado.

\begin{tabular}{|c|c|c|c|c|c|}
\hline \multirow{3}{*}{$\begin{array}{l}\text { Faixa } \\
\text { etária }\end{array}$} & \multirow{3}{*}{$\begin{array}{c}\text { Xenos } \\
\text { realizados }\end{array}$} & \multicolumn{4}{|c|}{ Xenos positivos } \\
\hline & & \multicolumn{2}{|c|}{ clássico* } & \multicolumn{2}{|c|}{ artificial $^{\star \star}$} \\
\hline & & $\mathrm{n}^{\circ}$ & $\%$ & $\mathrm{n}^{\circ}$ & $\%$ \\
\hline $7-30$ & 13 & 4 & 30,7 & 7 & 53,8 \\
\hline $31-60$ & 36 & 9 & 25,0 & 10 & 27,7 \\
\hline $61-80$ & 8 & 3 & 37,5 & 3 & 37,5 \\
\hline Total & 57 & 16 & 28,0 & 20 & 35,0 \\
\hline
\end{tabular}

${ }^{*}$ Fisher $p=0,53{ }^{\star \star}$ Fisher $p=0,101$.

Tabela 6 - Quantidade de triatomíneos aplicados e examinados em ambos os métodos.

\begin{tabular}{lccc}
\hline \multirow{3}{*}{ Método } & \multicolumn{3}{c}{ Triatomíneos } \\
\cline { 2 - 4 } & aplicados & \multicolumn{2}{c}{ examinados } \\
& $\mathrm{n}^{\circ}$ & $\mathrm{n}^{\circ}$ & $\%$ \\
\hline Clássico & 2280 & 2036 & $89^{*}$ \\
Artificial & 2280 & 1993 & $87^{\star}$ \\
\hline $\mathrm{p}=0,62$ & & &
\end{tabular}

$(2,6 \%)$ por $3,11(2,6 \%)$ por 2 e $11(2,6 \%)$ por apenas um triatomíneo. No xenodiagnóstico artificial, os 1993 triatomíneos examinados resultaram em 423 "pools" sendo $379(89,6 \%)$ constituídos de 5 triatomíneos, 7 (1,7\%) por 4, 9
$(2,1 \%)$ por $3,15(3,5 \%)$ por 2 e $13(3,1 \%)$ por apenas um triatomíneos (Tabela 7). Para ambos os métodos, a média de pools examinados por xenodiagnóstico foi $7( \pm 1)$

Grupo controle. Os 21 indivíduos do grupo controle apresentaram os xenodiagnósticos artificiais negativos.

Sorologia de controle. A sorologia de controle realizada no grupo chagásico pelas técnicas de HAI, IFI, e ELISA, confirmaram a positividade da sorologia prévia ao estudo, exceto em um caso. Este caso era um indivíduo do sexo feminino que tinha sorologia previa 
Tabela 7 - Distribuição do número e percentual de "pools" examinados nos xenodiagnósticos clássico e artificial, segundo a quantidade de triatomíneos por pool.

\begin{tabular}{|c|c|c|c|c|}
\hline \multirow{3}{*}{ № triatomíneos por "pool" } & \multicolumn{4}{|c|}{ Pools } \\
\hline & \multicolumn{2}{|c|}{ Xeno clássico } & \multicolumn{2}{|c|}{ Xeno artificial } \\
\hline & $\mathrm{n}^{\circ}$ & $\%$ & $\mathrm{n}^{\circ}$ & $\%$ \\
\hline 5 & 383 & 89,9 & 379 & 89,6 \\
\hline 4 & 10 & 2,3 & 7 & 1,7 \\
\hline 3 & 11 & 2,6 & 9 & 2,1 \\
\hline 2 & 11 & 2,6 & 15 & 3,5 \\
\hline 1 & 11 & 2,6 & 13 & 3,1 \\
\hline Total & 426 & 100,0 & 423 & 100,0 \\
\hline
\end{tabular}

positiva pelas técnicas de IFI e HAI, era procedente de área endêmica, com forte epidemiologia e queixas de palpitação e obstipação. Os dois pacientes admitidos com base na epidemiologia e na clínica de megaesôfago, apresentaram sorologia positiva. No grupo controle a sorologia de controle realizada pelas mesmos métodos e no mesmo laboratório foi negativa.

\section{DISCUSSÃO}

O xenodiagnóstico clássico tem sido aplicado há mais de 80 anos e tem dado grande contribuição ao diagnóstico e à pesquisa da doença de Chagas. Porém, as reações alérgicas ocasionais, decorrentes de sua aplicação, mostram a necessidade de métodos alternativos para algumas situações da doença de Chagas na atualidade. Um desses métodos tem sido o xenodiagnóstico artificial. Desde que foi proposto em 19471011 o método artificial tem sido comparado com o clássico e apresentado resultados similares 379 . Todavia, o xeno artificial nunca foi implementado. Provavelmente, a falta de uma membrana apropriada e de um anticoagulante adequado, dificultaram o seu uso mais sistemático. Somente nestas últimas décadas o método artificial tem despertado interesse, inicialmente devido aos transplantes de órgãos e, posteriormente, com o advento da SIDA2 17.

Neste trabalho, ambas as modalidades de xenodiagnóstico foram eficazes em demonstrar a parasitemia pelo $T$. cruzi. Os exames realizados simultaneamente, mostram a capacidade de ambos em captar o $T$. cruzi no sangue extraído de locais distintos, como o braço, para o xeno artificial, e a panturrilha, para o xeno clássico. Dentre os 24 indivíduos xenopositivos, metade foi positiva simultaneamente por ambos os métodos. A xenopositividade de $28 \%$ pelo método clássico e $35 \%$ pelo artificial, encontrase dentro da faixa de sensibilidade para o xenodiagnóstico e concorda com dados já publicados 1215 . Considerando os resultados relatados em outros trabalhos 1417 , a positividade acumulada de $42 \%$ por ambos os métodos foi boa. Não conseguiu-se mostrar a superioridade do xenodiagnóstico artificial encontrada por Santos et al14. Embora o xeno artificial tendesse a mostrar maior número de pessoas xenopositivas, não houve diferença estatisticamente significante.

O xenodiagnóstico artificial tem a vantagem de ser mais confortável para o paciente, elimina eventuais reações alérgicas decorrentes da picada do triatomíneo e permite repetições do exame com maior facilidade. O xeno artificial é mais apropriado para o chagásico imunossuprimido, diabético, canceroso, leucêmico e transplantado. Nestes pacientes, quando indicado, seria preferível aplicar o xenodiagnóstico artificial, não porque os triatomíneos ao picarem a pele transmitam infecções, mas porque a lesão da pele seja pela picada, ou pelo trauma ao coçar, pode facilitar a penetração de bactérias com conseqüências negativas, especialmente para os pacientes neutropênicos.

O número de triatomíneos que mudaram para $\circ 2^{\circ}$ estágio foi similar em ambos os métodos. A média de pools por xeno em ambos os métodos foi $7( \pm 1)$. Os pools com 5 triatomíneos representaram $89,9 \%$ e $86,6 \%$ nos xenos clássico e artificial, respectivamente.

A utilidade prática deste aparelho portátil de xenodiagnóstico artificial, no campo, teve algumas limitações. No posto médico de Mambaí funcionou bem, mas o transporte do aparelho de casa em casa no interior do município apresentou dificuldades pela falta de energia elétrica, 
ausência de uma mesa onde coloca-lo e sua montagem e desmontagem em cada residência. Estas dificuldades limitam a prática do xenodiagnóstico artificial com este aparelho em áreas remotas, onde o xeno clássico pode ser realizado facilmente por um agente de saúde. Para ser útil, o aparelho deve permanecer fixo em um posto de saúde e os pacientes se deslocarem para o local, o que traz dificuldades para quem reside a vários quilômetros de distância. Dos três indivíduos da zona rural de Mambaí, nos quais foi colhido sangue e realizado o xeno artificial quatro horas após, dois foram negativos e um foi positivo nos dois métodos, com cinco pools positivos em sete examinados no xeno clássico e três pools positivos em oito examinados no artificial. Este dado mostra que o intervalo de quatro horas entre a coleta de sangue e a realização do xeno artificial, não prejudica a positividade do exame.

O xeno artificial é prático porém é mais trabalhoso do que o clássico, requerendo cuidado ao manusear sangue e habilidade para punção venosa. Em duas ocasiões houve rotura do preservativo com exposição do sangue, sendo portanto necessário trabalhar com óculos, jaleco e luvas. O xeno clássico é mais simples, pode ser aplicado por um agente de saúde na residência do indivíduo, ou mesmo durante sua jornada de trabalho na lavoura. Para o xeno artificial, o paciente colhe o sangue e pode ser dispensado, enquanto no xeno clássico ele deve permanecer entre 30 e 60 minutos realizando o exame. No xeno artificial, o sangue deve estar na temperatura apropriada para que os triatomíneos suguem.

No curso do estudo, $5(9 \%)$ indivíduos apresentaram reações alérgicas decorrentes da aplicação do xeno clássico. Em dois, as reações foram imediatas à retirada dos triatomíneos e se apresentaram como eritema e pápulas pruriginosas com evolução autolimitada. Nos três restantes, as reações ocorreram cinco, sete e 11 dias após o exame, apresentando prurido e pápulas enduradas. Um destes, que fez - exame pela primeira vez, usou corticóide tópico. As reações tardias têm sido descritas 48 a 72 horas após o exame 6813 . A ocorrência de reações tardias tem importância no trabalho de campo porque é freqüente pesquisadores aplicarem o xenodiagnóstico e logo voltarem às instituições de origem, ignorando a possibilidade de ocorrerem reações cutâneas seis ou oito dias após o exame. Essas manifestações iatrogênicas contra-indicam a repetição do xenodiagnóstico clássico e reforçam a utilidade do xeno artificial.

\section{REFERÊNCIAS BIBLIOGRÁFICAS}

1. Brumpt E. O xenodiagnostico. Aplicação ao diagnostico de algumas infecções parasitárias e em particular à trypanosomose de Chagas. Annaes Paulistas de Medicina e Cirurgia 3:97-102, 1914.

2. Campos R, Amato Neto V, Matsubara L, Moreira AAB, Pinto PLS. Estudos sobre o xenodiagnóstico "in vitro". 1. Escolha de anticoagulante e de membrana. Revista do Hospital das Clinicas da Faculdade de Medicina de São Paulo 43:101-103, 1988.

3. Carvalho G. Método indireto para alimentação de insetos hematófagos. Revista Brasileira de Biologia 21:193-196, 1961.

4. Costa CHN, Costa MT, Weber JN, Gilks GF, Castro C, Marsden PD. Skin reactions to bugs bites as a result of xenodiagnosis. Transactions of the Royal Society of Tropical Medicine and Hygiene 75:405-408, 1981.

5. Dias E. Técnica do xenodiagnóstico na moléstia de Chagas. Memórias do Instituto Oswaldo Cruz 35:335-344, 1940.

6. Dias JCP. Manifestações cutâneas na prática do xenodiagnóstico. Revista Brasileira de Malariologia e Doenças Tropicais 20:248-257, 1968.

7. Freitas JLP, Nussenzweig V, Amato Neto V, Sonntag R. Estudo comparativo entre xenodiagnósticos praticados "in vivo" e "in vitro" em formas crônicas de moléstia de Chagas. O Hospital 17:101-110, 1955.

8. Mott KE, França JT, Barrett TV, Hoff R, Oliveira TS, Sherlock IA. Cutaneous allergic reactions to Triatoma infestans after xenodiagnosis. Memórias do Instituto Oswaldo Cruz 75:3-10, 1980.

9. Nussenzweig V, Sonntag R. Xenodiagnóstico artificial. Novo processo. Primeiros resultados positivos. Revista Paulista de Medicina 40:41-43, 1952.

10. Romaña C. Enfermedad de Chagas. Lopez Libreros Editores, Buenos Aires, p. 88, 1963.

11. Romaña C, Gil J. Xenodiagnóstico artificial. Anales del Instituto de Medicina Regional (Tucuman) 2:5760, 1947.

12. Salgado AA. Consideraciones sobre metodologia y sensibilidad del xenodiagnostico. Boletin Chileno de Parasitologia 24:9-13, 1969.

13. Salgado JA, Salgado AA, Espinola HN. Contribuição ao conhecimento das reações às picadas de triatomíneos. Revista Brasileira de Malariologia e Doenças Tropicais 20:231-235, 1968.

14. Santos AH, Silva IG, Rassi A. Estudo comparativo 
entre o xenodiagnóstico natural e o artificial, em chagásicos crônicos. Revista da Sociedade Brasileira de Medicina Tropical 28:367-373, 1995.

15. Schenone H, Alfaro E, Reyes H, Taucher E. Valor del xenodiagnostico en la infeccion chagasica cronica. Boletin Chileno de Parasitologia. 23: 149-154, 1968.

16. Silva IG. Dispositivo para realização do xenodiagnóstico artificial. Revista de Patolgia Tropical 20:35-38, 1991.

17. Souza HBWT, Moreira AAB, Matsubara L, Campos R, Amato Neto V, Pinto PLS, Takiguti CK. Estudo sobre o xenodiagnóstico "in vitro". II - comparação com o xenodiagnóstico "in vivo". Revista do Hospital das Clínicas da Faculdade de Medicina de São Paulo 43:165- 167, 1988.

18. Tarello, R. Torrealba. La Nueva Grafica p. 7-19 , 1964 Torino, Italia.

19. Torrealba JF. Investigaciones sobre la enfermedad de Chagas en San Juan de los Morros. Caracas Imprenta Nacional, p. 418-419, 1958. 